\begin{abstract}
PARKINSON's disease (PD) is an extra-pyramidal neurodegenerative disorder, in which alterations of the immune system are involved. Interleukin (IL)-15 stimulates cellular immune response and induces growth and differentiation of various immune cells. RANTES, promoting leukocyte infiltration to sites of inflammation, mediates the trafficking and homing of immune cells. To clarify the potential effect of levodopa on the immunological network of PD, we analyzed IL-15 and RANTES serum levels in PD patients, treated or not with levodopa, and in healthy donors. Levodopa-treated patients showed significantly higher IL-15 and RANTES circulating levels with respect to healthy controls and higher, although not significantly, levels with respect to untreated patients. So, we hypothesize that the immunological alterations found in PD may be linked, at least in part, to levodopa therapy.
\end{abstract}

Key words: interleukin-15, RANTES, Parkinson's disease, levodopa

\section{Effect of levodopa on interleukin- 15 and RANTES circulating levels in patients affected by Parkinson's disease}

\author{
Sebastiano Gangemi ${ }^{1, C A}$, Giorgio Basile ${ }^{2}$, \\ Rosaria Alba Merendino ${ }^{3}$, Antonio Epifanio ${ }^{4}$, \\ Giuseppe Di Pasquale ${ }^{5}$, Benito Ferlazzo ${ }^{1}$, \\ Vittorio Nicita-Mauro ${ }^{2}$ and Letterio Morgante ${ }^{4}$
}

${ }^{1}$ School and Division of Allergy and Clinical Immunology, Via Centonze n. 200 is. 98, 98123 Messina,italy; ${ }^{2}$ School and Division of Geriatric Medicine; ${ }^{3}$ Chair of Immunopathology, Department of Human Pathology; ${ }^{4}$ Department of Neuroscience and ${ }^{5}$ Department of Pediatric Science and Medical Surgery, University of Messina, Messina, Italy

\footnotetext{
${ }^{\mathrm{CA}}$ Corresponding author

Tel: + 39902212096 ;

Fax: + 39906782336 ;

E-mail: sgangemi@eniware.it
}

Parkinson's disease (PD) is an extra-pyramidal neurodegenerative disorder characterized by a decreased number of nigrostriatal dopaminergic neurons, in which alterations of the immune system have been postulated to be one of the mechanisms underlying the pathogenesis. ${ }^{1}$ In particular, alteration of cytokine production, such as interleukin (IL)-6, by brain and peripheral blood cells in PD patients was found. ${ }^{2}$

IL-15, a monocyte and epithelial cell-derived cytokine, produced in response to environmental stimuli and infectious agents, shares a variety of biological functions including stimulation and maintenance of the cellular immune response. ${ }^{3}$ This cytokine, similar to IL-2 in receptor specificity and biological functions (the specificity for IL-15 is provided by unique private $\alpha$-chain receptors), is important for the growth and differentiation of $\mathrm{B}$ lymophocytes and T lymphocytes, natural killer cells cells, macrophages and monocytes; and, consequently, its pleiotropic activity results in immunoregulatory cross-talk between natural and specific immune cells. ${ }^{3}$

RANTES, a pro-inflammatory chemokine, is a small protein of 68 amino acids that induces leukocyte migration and mediates the trafficking and homing of classical lymphoid cells such as T cells and mono- cytes, but also acts on a range of other cells, including basophils, eosinophils, natural killer cells, dendritic cells and mast cells ${ }^{4}$ RANTES acts by promoting leukocyte infiltration to sites of inflammation. ${ }^{4}$

Since, at present, there are no data regarding IL-15 and RANTES serum levels in parkinsonian patients, in order to clarify the role of these mediators and the effect of levodopa in the pathogenesis of PD we analyzed IL-15 and RANTES serum levels in untreated and in levodopa-treated PD patients, comparing the results with a group of healthy donors.

Twenty-four patients affected by idiopathic PD, in accordance with Gelb criteria, were consecutively recruited: 12 patients (eight male, four female) received 1-dopamine therapy (group A) and 12 patients (seven male, five female) were free from any specific anti-parkinsonian treatment (group B). In group A the mean age was 65.15 years (range, 5475 years), the mean l-dopamine dosage was $675 \pm$ $180 \mathrm{mg} /$ day, the mean Hoehn and Yahr stage was $3.1 \pm 0.5$, and the mean duration of illness was $84 \pm 23$ months. In group $\mathrm{B}$, the mean age was 58.4 years (range, 45-77 years), mean Hoehn and Yahr stage was $1.3 \pm 0.2$, and the mean duration of illness was $26 \pm 10$ months. Twelve healthy donors (group C), sex and age matched, were enrolled as controls. 
Each patient and subject gave written informed consent.

A $10 \mathrm{ml}$ blood sample was collected from the antecubital vein, allowed to clot at room temperature for $2 \mathrm{~h}$, separated by centrifugation at $200 \times \mathrm{g}$ for 15 min in a 4235 A centrifuge (ALC Int. S.r.L., Milan, Italy) and stored at $-80^{\circ} \mathrm{C}$ until use.

Serum IL-15 and RANTES levels were assayed by immunoenzymatic methods (Quantikine Human IL15 and Human RANTES ELISA kits; R\&D System, SPACE Import-Export, Milan, Italy). The minimum detectable dose of IL-15 and RANTES is $<2 \mathrm{pg} / \mathrm{ml}$ and $8 \mathrm{pg} / \mathrm{ml}$, respectively.

Differences in serum levels were assessed by oneway analysis of variance and the Student-NewmanKeults test. Data were expressed as the mean \pm standard deviation. $p<0.05$ was considered significant.

Correlation coefficients were calculated with the Spearman rank test. All data were analyzed using the STAT VIEW SE program (Abacus, Berkeley, CA, USA).

This preliminary study shows normal values of circulating IL-15 and RANTES in untreated PD patients, confirming a different involvement of the various mediators in the ambit of PD (Fig. 1A,B).

For example, Bessler et al. showed that the production of IL-2 by peripheral blood mononuclear cells (PBMC) from PD patients was significantly lower with respect to healthy controls, whereas the secretion of IL-1 $\beta$, IL-6 and TNF- $\alpha$ was significantly enhanced. ${ }^{1}$
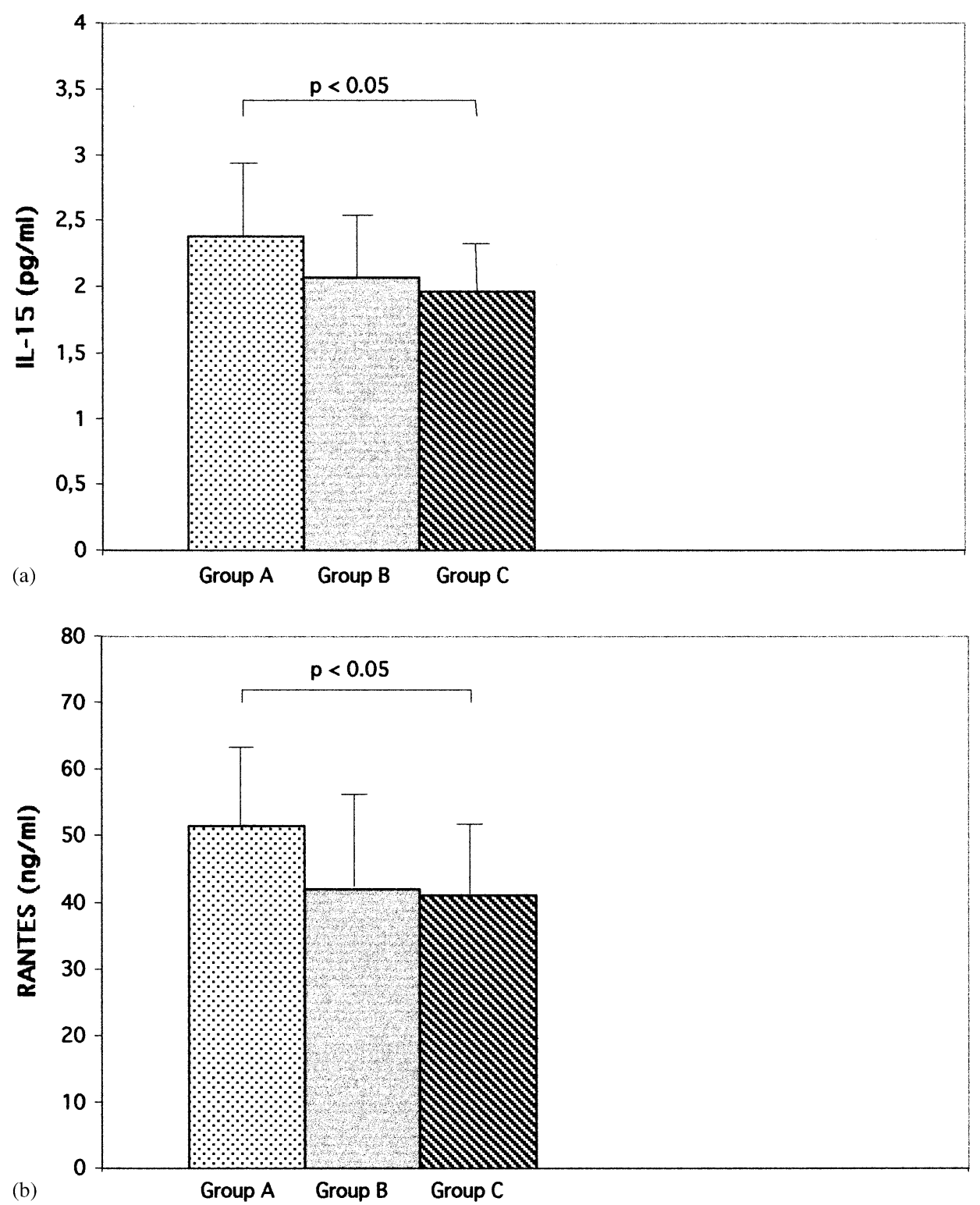

FIG. 1. (A) IL-15 serum levels in patients with Parkinson's disease treated with levodopa (group A), in untreated PD patients (group B) and in healthy controls (group C). (B) RANTES serum levels in patients with PD treated with levodopa (group A), in untreated PD patients (group B) and in healthy controls (group C). 
In addition, IL-15 and RANTES serum levels from levodopa-treated PD patients were significantly higher with respect to those from healthy controls and higher, although not significantly, with respect to those from untreated PD patients (Fig. 1A,B).

In previous papers, it has been demonstrated that levodopa exerts an immuno-modulatory effect on PBMC from PD patients, with an enhancement of IL-6 and TNF- $\alpha$ production by PBMC from PD patients. ${ }^{1}$

Even if no correlation between IL-15 or RANTES and stage or duration of disease among our patients was found, the possibility that disease stage may contribute to the enhanced expression of these cytokines cannot be excluded.

So we hypothesize, in agreement with other authors, that the immunological alterations observed in levodopa-treated patients might be linked, both to levodopa treatment and to disease progression.

Furthermore, the contemporaneous increase of circulating IL-15 and RANTES among patients with advanced stage of disease and severe functional impairment may reflect the tight link between these mediators.

In fact, an important action of IL-15 is the induction of CC-type, CXC-type and C-type chemokines in T lymphocytes and CC chemokine receptors, such as RANTES and MIP- $-\alpha_{\alpha}{ }^{5}$

Normal human microglia expresses constitutively mRNA transcripts for IL-15 and for IL-15R. ${ }^{3}$ Activated microglia in neurological diseases such as Alzheimer disease, amyotrophic lateral sclerosis, stroke or PD can overproduce mediators of phlogosis and activate peripheral metabolic, endocrine and immune responses. $^{2}$

Our results on IL-15 and RANTES circulating levels strengthen the data about the existence of a tight connection between the central nervous system and the immune system.

The increase of IL-15 production may enhance the further recruitment of inflammatory cells in brain by RANTES induction, so increasing the cerebral inflammation.

In conclusion, this study may contribute to a better definition of immunological network involved in PD.

ACKNOWLEDGEMENTS. This work was supported in part by a grant from the University of Messina Medical School (ex 60\%), Italy.

\section{References}

1. Bessler H, Djaldetti R, Salman H, Bergman M, Djaldetti M. IL-1(, IL-2, IL-6 and TNF-( production by peripheral blood mononuclear cells from patients with Parkinson's disease. Biomed Pharmacother 1999; 53: 141 145

2. Terreni L, De Simoni MG. Role of the brain in interleukin-6 modulation Neuroimmunomodulation 1998; 5: 214-219.

3. Fehniger TA, Caligiuri MA. Interleukin-15: biology and relevance to human disease. Blood 2001; 97: 14-32.

4. Appay V, Rowland-Jones SL RANTES. a versatile and controversial chemokine. Trends Immunol 2001; 22: 83-87.

5. Perera LP, Goldman CK, Waldmann TA. IL-15 induces the expression of chemokines and their receptors in T lymphocytes. J Immunol 1999; 162: $2606-2612$.

\section{Received 2 May 2003 \\ Accepted 2 June 2003}




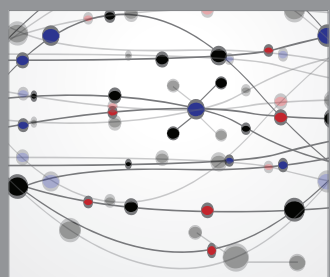

The Scientific World Journal
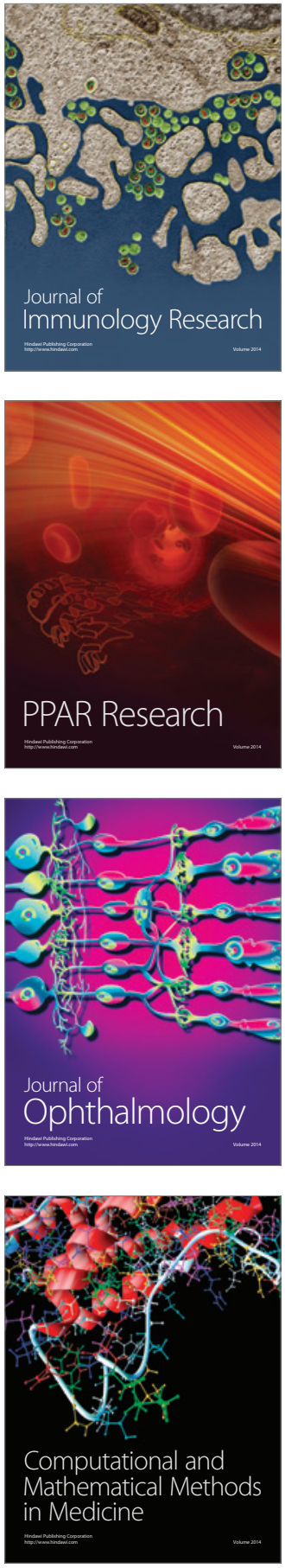

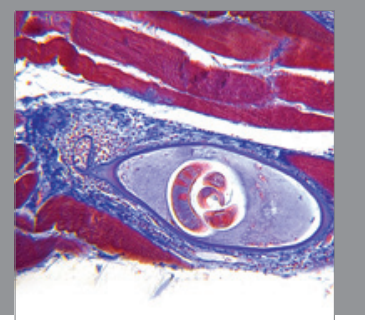

Gastroenterology

Research and Practice
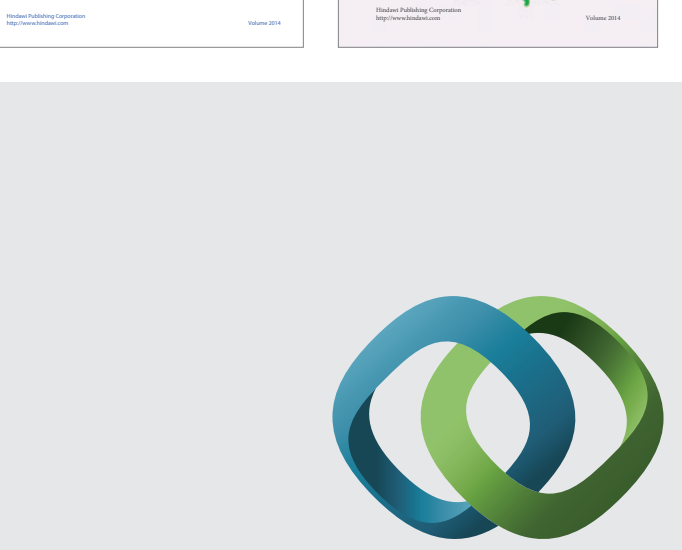

\section{Hindawi}

Submit your manuscripts at

http://www.hindawi.com
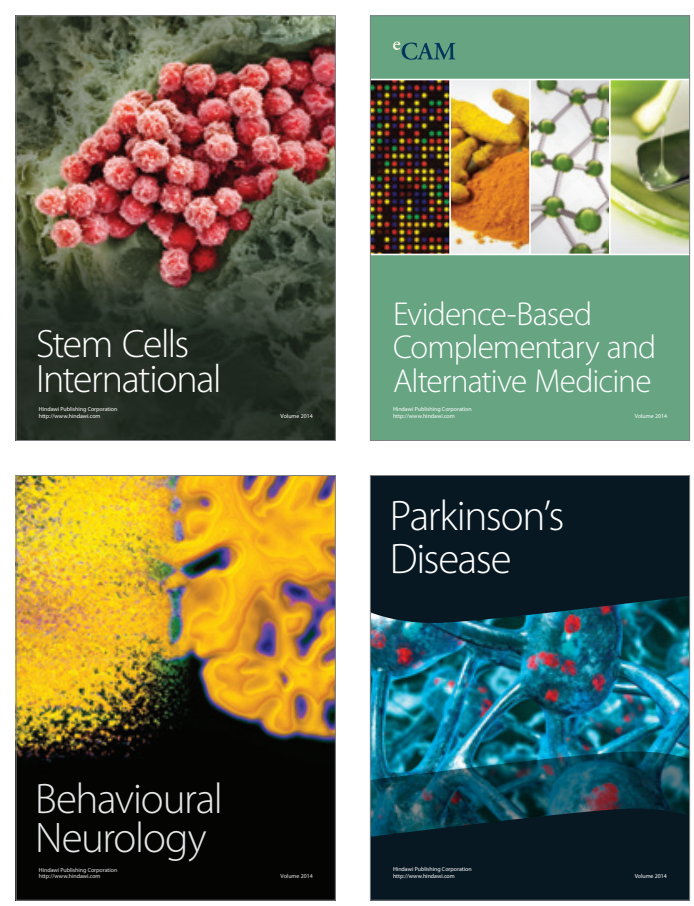

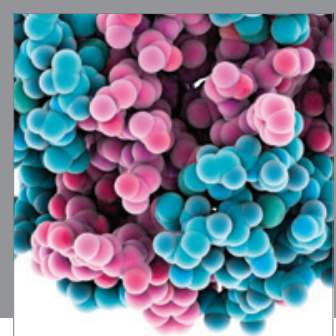

Journal of
Diabetes Research

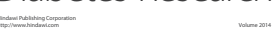

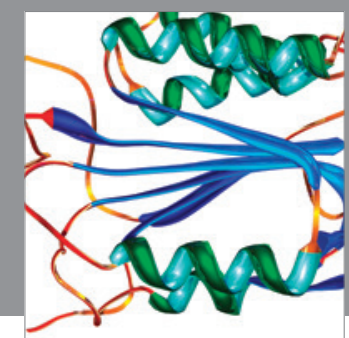

Disease Markers
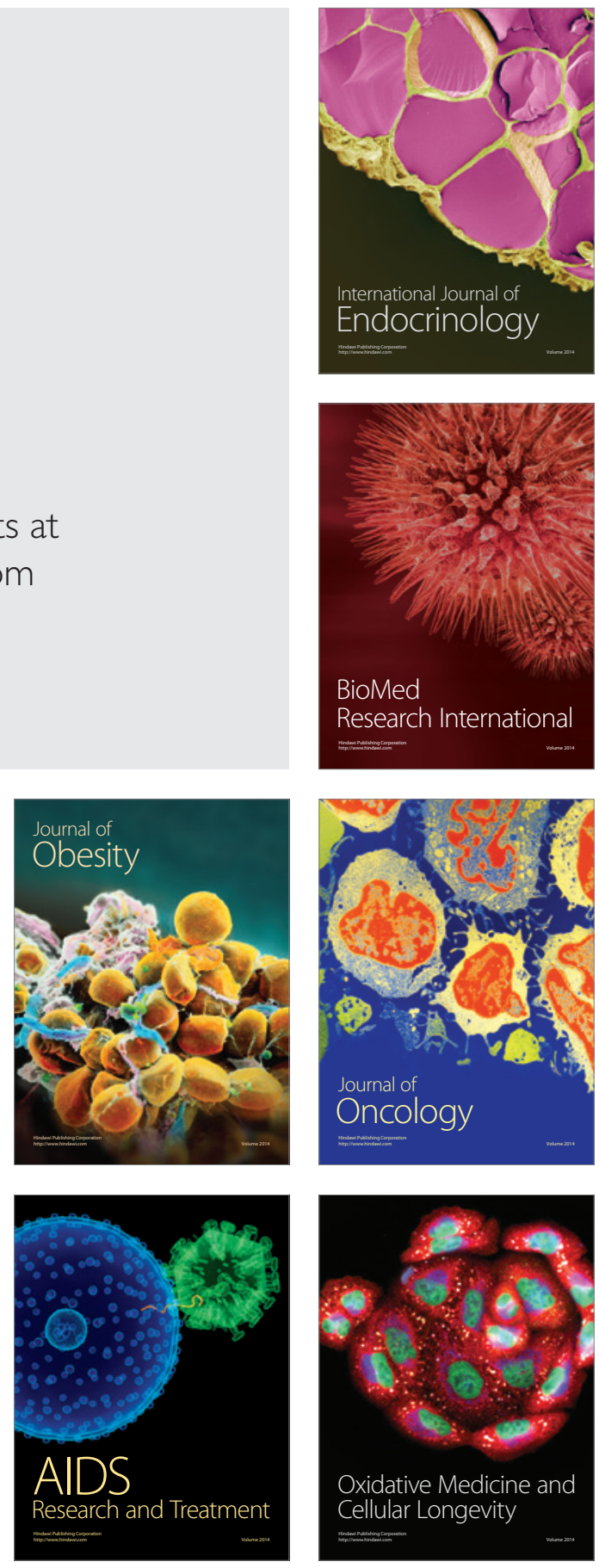